\title{
3D-printed individualized tooth-borne tissue retraction devices compared to conventional dental splints for head and neck cancer radiotherapy: a randomized controlled trial
}

Thomas Held ${ }^{1,2,3^{*}}$ (D) Christopher Herpel ${ }^{7}$, Franz Sebastian Schwindling ${ }^{7}$, Leo Christ ${ }^{1,2}$, Kristin Lang $^{1,2,3}$, Sebastian Regnery ${ }^{1,2,3}$, Tanja Eichkorn 1,2,3, Adriane Hommertgen ${ }^{1,2}$, Cornelia Jaekel ${ }^{1,2}$, Johannes Krisam ${ }^{8}$, Julius Moratin ${ }^{9}$, Jan Mrosek ${ }^{9}$, Karl Metzger ${ }^{9}$, Karim Zaoui ${ }^{10}$, Tracy Moutsis ${ }^{10}$, Semi Harrabi 1, 2,3,4,5,6, Klaus Herfarth 1,2,3,4,5,6, Christian Freudlsperger ${ }^{9}$, Peter Rammelsberg ${ }^{7}$, Jürgen Debus , $^{1,2,3,4,5,6}$ and Sebastian Adeberg $1,2,3,4,5,6$

\begin{abstract}
Background: Despite modern treatment techniques, radiotherapy (RT) in patients with head and neck cancer (HNC) may be associated with high rates of acute and late treatment-related toxicity. The most effective approach to reduce sequelae after RT is to avoid as best as possible healthy tissues and organs at risk from the radiation target volume. Even small geometric changes can lead to a significant dose reduction in normal tissue and better treatment tolerability. The major objective of the current study is to investigate 3D printed, tooth-borne tissue retraction devices (TRDs) compared to conventional dental splints for head and neck RT.

Methods: In the current two-arm randomized controlled phase II trial, a maximum of 34 patients with HNC will be enrolled. Patients will receive either TRDs or conventional dental splints (randomization ratio 1:1) for the RT. The definition of the target volume, modality, total dose, fractionation, and imaging guidance is not study-specific. The primary endpoint of the study is the rate of acute radiation-induced oral mucositis after RT. The quality of life, local control and overall survival 12 months after RT are the secondary endpoints. Also, patient-reported outcomes and dental status, as well as RT plan comparisons and robustness analyzes, will be assessed as exploratory endpoints. Finally, mesenchymal stem cells, derived from the patients' gingiva, will be tested in vitro for regenerative and radioprotective properties.
\end{abstract}

Discussion: The preliminary clinical application of TRD showed a high potential for reducing acute and late toxicity of RT in patients with HNC. The current randomized study is the first to prospectively investigate the clinical tolerability and efficacy of TRDs for radiation treatment of head and neck tumors.

Trial registration: ClinicalTrials.gov; NCT04454697; July $1^{\text {st }}$ 2020; https://clinicaltrials.gov/ct2/show/record/NCT04 454697.

*Correspondence: thomas.held@med.uni-heidelberg.de

1 Department of Radiation Oncology, Heidelberg University Hospital, Im

Neuenheimer Feld 400, 69120 Heidelberg, Germany

Full list of author information is available at the end of the article

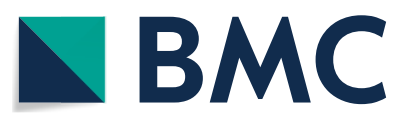

(c) The Author(s) 2021. Open Access This article is licensed under a Creative Commons Attribution 4.0 International License, which permits use, sharing, adaptation, distribution and reproduction in any medium or format, as long as you give appropriate credit to the original author(s) and the source, provide a link to the Creative Commons licence, and indicate if changes were made. The images or other third party material in this article are included in the article's Creative Commons licence, unless indicated otherwise in a credit line to the material. If material is not included in the article's Creative Commons licence and your intended use is not permitted by statutory regulation or exceeds the permitted use, you will need to obtain permission directly from the copyright holder. To view a copy of this licence, visit http://creativecommons.org/licenses/by/4.0/. The Creative Commons Public Domain Dedication waiver (http://creativecommons.org/publicdomain/zero/1.0/) applies to the data made available in this article, unless otherwise stated in a credit line to the data. 
Keywords: Intensity-modulated radiotherapy, Particle therapy, Toxicity, Radiation-induced oral mucositis, Xerostomia, Squamous cell carcinoma, Salivary gland tumor

\section{Background}

Despite modern radiation techniques, e.g. intensitymodulated radiotherapy (IMRT), radiation treatment may be associated with high rates of severe treatmentrelated toxicity $[1,2]$. Higher grade radiation-induced oral mucositis (RIOM) ( $\geq$ Common Terminology Criterion $[\mathrm{CTC}]$ grade III), common dose-limiting toxicity, occurs in up to $60 \%$ of patients [3-5]. The rate of RIOM is further increased by the application of simultaneous systemic therapies (e.g., chemotherapy). The immediate consequences of this are limited tolerability of therapy and reduced compliance, and an interruption or even discontiuation of treatment [6]. If epitheliolysis and ulcerations occur, the risk of bacterial superinfection with possible complications, including sepsis, increases. In the long term, mucositis causes dry mouth, taste disorders, pain, and difficulty swallowing, and weight loss, which increases the frequency of hospitalization. Many prospective clinical studies demonstrated a reduction in the quality of life in this context $[7,8]$.

The probability of radiogenic damage to epithelial cells is dose-dependent [9]. Established risk factors for the development of oral mucositis during radiotherapy (RT) include decreased oral hygiene and smoking. So far, many approaches have been postulated for the prevention and treatment of mucositis, but there are no clear recommendations other than adequate care and analgesia. A total of 320 studies listed about oral mucositis at "http://www. clinicaltrials.gov" underscore the clinical relevance of the topic.

However, the most effective approach to reducing acute toxicity after RT is to avoid as best as possible healthy tissues and organs at risk from the high-dose area of the radiation field. Isolated case reports [10] and several retrospective studies [11-13] on customized TRDs for RT of head and neck tumors reported reduced rates of mucositis with good tolerability. Currently, there are no prospective studies available.

Usually, there is a close positional relationship in the head and neck area between the gross tumor volume and the adjacent normal tissue and organs at risk. Due to anatomical reasons, including parts of the tongue, jaw, or oral mucosa in the high-dose RT area is often unavoidable. Using TRDs can increase the distance between the tumor and normal tissue in certain areas. Even the smallest geometric change of a few millimeters can significantly reduce the dose of normal tissue with significant benefits for the patient [14]. This is essential in particular for high precision RT, e.g. by IMRT, MRI-guided RT, or heavy ion therapy. Another factor of uncertainty is the mobility of the tongue and the base of the tongue because there is usually no fixation during RT. Proper immobilization can reduce unwanted movements and allow for more precise therapy. Besides increasing the distance between the tumor and healthy tissue to reduce RIOM, TRDs could also be advantageous for precise repositioning of the patient.

Traditionally, manufacturing TRDs that target healthy tissue displacement, tongue immobilization, and precise patient repositioning is complex and time-consuming [15]. This could be the reason conventional dental splints, made of thermoplastic resin, are used as the standard of clinical treatment in many institutions. Therefore, these thermoplastic dental splints will be used in the control arm of the present study. Introducing novel computerassisted design and manufacturing techniques, e.g. 3D printing, has the potential to simplify TRD's workflow while reducing costs and increasing the quality of TRD. For certain indications, e.g. craniomandibular disorders, the production of aligners and TRDs using the 3D printing process is already established in our institution [16, 17]. The materials and methods used are approved and tested for intraoral use. The 3D printing process enables economical and flexible production.

The current trial aims to evaluate TRDs for individualized and effective RT of patients with malignant HNC to protect normal tissue and improve treatment tolerability. At the cellular level, the high regenerative potential of mesenchymal stem cells (MSCs) has been characterized in detail [18]. They can be isolated from various tissues such as bone marrow and adipose tissue, but also the head and neck region (including the gingiva). The importance of gingival MSCs for radioprotection in the head and neck area has not been elucidated.

\section{Methods/design}

The current single-center, two-arm, randomized controlled phase II trial anticipates an enrollment of a maximum of 34 patients who meet the inclusion criteria. Patients will receive 3D printed dental TRDs or conventional dental splints (in a randomization ratio of 1:1 via block randomization using an online randomization tool). TRDs are pre-fabricated using 3D printing and are tailored to the individual patient and treatment intention. Each TRD comprises a fixation piece on the tooth which allows the opening of the mouth and the mandibular 
protrusion, the reproducible positioning, and the covering of the teeth in combination with lip- and cheek spacing. Also, a retraction part allows the displacement of the tongue. An example of a clinical case is shown in Fig. 1. A publication summarizing details of the design process and TRD fabrication is currently under preparation. Conventional dental splints are protective plastic stents covering the teeth to reduce the radiation overdose caused by dental restoration materials. No additional retraction parts or spacing devices are attached.

About $50-70 \%$ of patients require tooth extractions, e.g. due to caries, before RT [19]. In general, small amounts of periodontal tissue remain attached to the surface of the extracted teeth. From these tissue probes, isolation and expansion of the gingival MSCs will be performed. After isolation and expansion, clonogenicity and proliferation are examined in cell culture before and after in vitro radiation. Also, functional properties such as cell adhesion and migration are characterized, and the capacity for differentiation. Regenerative and radioprotective factors eventually correlate with the systematically recorded toxicity profile of patients.
To evaluate the consequences of TRDs on the integral dose to adjacent normal tissue, plan comparisons will be made between all patients in both treatment groups. In selected patients with available pre-treatment diagnostic computed tomography $(\mathrm{CT})$ in the experimental arm, in silico intra-patient plan comparisons will further clarify the potential clinical benefits of TRDs concerning dose distribution. The robustness of TRD treatment plans concerning positional variability will be assessed as part of the routine daily imaging guide based on cone beam $\mathrm{CT}$ or orthogonal X-rays during treatment. The mean displacement of osseous reference points in the upper and lower jaw will be compared between both groups to assess the maxillomandibular position changes during RT. The total duration of the study is scheduled to be 36 months, including a 24-month recruitment phase and a minimum follow-up of 12 months.

\section{Inclusion criteria}

The inclusion criteria are: diagnosed malignant tumors in the head and neck region; clinical target volume includes parts of one or several of the following anatomical

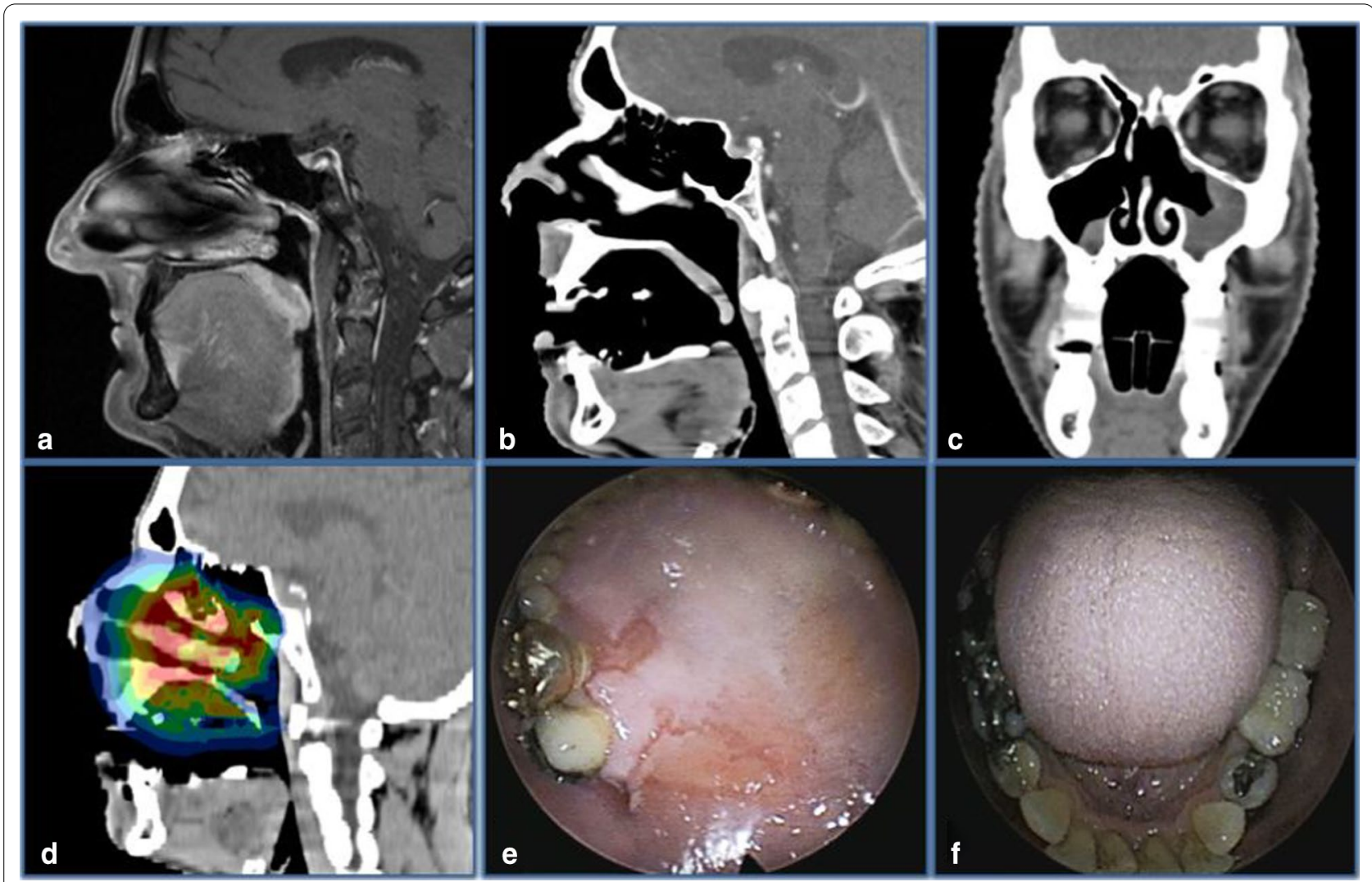

Fig. 1 a T1 contrast-enhanced fat-saturated magnetic resonance imaging of a patient with sarcoma of the paranasal sinuses without tissue retraction device (TRD). b, c Contrast-enhanced computed tomography of the same patient with TRD. $\mathbf{d}$ Radiation treatment plan with TRD in situ. e Oral mucositis CTC grade III in the high-dose area of the radiation field. $\mathbf{f}$ Nonirritated tongue surface directly opposite the high-dose area of the radiation field 
regions: the upper jaw, the lower jaw, the hard palate, the tongue, the floor of the mouth, the buccal mucosa, the soft palate and the base of the tongue; an indication of adjuvant or definitive RT; age $\geq 18$ years; Karnofsky performance score $\geq 60$; complete wound healing after the surgical intervention; written informed consent; the ability of the subject to understand the character and individual consequences of the trial; for women of childbearing age (and men), adequate contraception.

\section{Exclusion criteria}

Exclusion criteria are: previous head and neck RT; multifocal diffuse tumor growth; patients who have not recovered from the acute toxicities of previous therapies; trismus (mouth opening $\leq 2 \mathrm{~cm}$ ); simultaneous chemo/ immunotherapy; evidence that the patient cannot adhere to the study protocol (e.g., non-compliance); the refusal of patients to participate in the study.

\section{Radiation therapy}

Radiation treatment will be carried out according to the current standard in our institution, following current clinical guidelines for HNC. The definition of the target volume, modality, total dose, fractionation, and imaging guidance is not study-specific. Simultaneous systemic therapies, particularly chemo- or immunotherapy, are not allowed in the current study. Due to the various histological entities projected for the current trial, a wide range of different treatment approaches is possible.

For treatment planning, patients will be immobilized with an individual immobilization mask. All patients will receive a non-contrast planning $\mathrm{CT}$ scan with a layer thickness of $3 \mathrm{~mm}$ and, if possible, also a contrastenhanced CT or magnetic resonance imaging (MRI) for an optimal definition of the target volume. Treatment planning will be carried out using Syngo PT-Planning (Siemens, Erlangen, Germany) and RayStation ${ }^{\circledR}$ (RaySearch Laboratories, Stockholm, Sweden) planning software. Automated multi-atlas-based segmentation of normal tissue and organs at risk, including salivary glands, tongue, hard and soft palate, and mandible, will be performed according to EORTC guidelines using the software RayStation ${ }^{\circledR}$. Dose constraints for normal tissues and organs will be respected according to the Quantitative Analyses of Normal Tissue Effects in the Clinic (QUANTEC) [20, 21]. According to standard procedures in our clinic, IMRT will be performed 5 fractions per week under image guidance with daily CT images and position correction using volumetric modulated arc therapy. If indicated, particle therapy will be done 5-6 fractions per week with protons or carbon ions. The active raster scanning technology will be used for the application of orthogonal X-ray image guidance and daily position correction. The target volume definition and the dose prescription will be left to the discretion of the treating radiation oncologist, following current clinical guidelines for $\mathrm{HNC}$ [22, 23].

\section{Study endpoints}

The primary endpoint of the study is the rate of acute RIOM after RT with TRD compared to conventional dental splints. To allow an objective evaluation of RIOM complementary to clinical questionnaires, the oral cavity and oropharynx are subdivided into eight anatomical regions (Table 1). Based on previous RIOM studies [3-5], we defined the assumed rate of severe oral mucositis ( $\geq$ CTC grade III) as $60 \%$ in the treated anatomical region and its direct proximity. The primary target value is to prevent severe RIOM ( $\geq$ CTC grade III) in the anatomical regions directly adjacent to the CTV in $75 \%$ of patients with TRD. To assess the primary endpoint, all patients receive a diagnostic video endoscopy of the oral cavity and oropharynx performed by an ENT specialist directly after the last radiation treatment. A blinded evaluation of the mucositis grade will be performed.

Quality of life, local control, and OS 12 months after RT are the secondary endpoints of the trial. After 12 months of study specific follow-up, the patients will continue with regular clinical follow-up examinations. Also, patient-reported outcomes (PRO) and dental status, as well as plan comparisons and robustness analyzes, will be assessed as exploratory endpoints. Also, the functional and differentiation parameters, as well as the regenerative and radioprotective factors of gingival MSCs will be evaluated in vitro.

\section{Study visits and evaluation criteria}

The follow-up corresponds to the clinical routine, except for the quality of life questionnaires, PRO, and dental status assessment. The Quality of Life Questionnaire (QLQ) of the European Organization for Research and Treatment of Cancer (EORTC) - H\&N35 [24], the Groningen Radiotherapy Induced Xerostomia questionnaire (GRIX) [25] and 12 HNC specific items from the CTC-PRO library [26] will be used in the current trial. The first study visit will be conducted 6 weeks

Table 1 Anatomical regions of the oral cavity and oropharynx

\begin{tabular}{ll}
\hline Oral cavity & Oropharynx \\
\hline Hard palate & Soft palate/uvula \\
Buccal mucosa right/left & Oropharyngeal sidewall right/left \\
Tongue (anterior 2/3) & The base of the tongue \\
Floor of mouth & Posterior pharyngeal wall \\
\hline
\end{tabular}

According to the 8th edition of the Union for International Cancer Control (UICC) tumor node metastasis (TNM) classification 
after RT, and thereafter every 3 months within the first year after treatment. Detailed information on study visits and evaluation criteria is shown in Fig. 2.

Patients will be followed for at least 12 months after RT to document any acute and subacute toxicity from CTC v5.0 that is related to study treatment. Response to treatment and progression will be defined according to the most recent Response Evaluation Criteria in Solid Tumors (RECIST) 1.1. Patients with partial follow-up are weighted by the proportion of the follow-up time that is completed.

\section{Data management and statistics}

The data is collected, managed, and processed electronically in the internal research database. The statistical analysis is based on the "Structure and Content of Clinical Study Reports" of the International Conference on Harmonization Guidelines and "Statistical Principles for Clinical Trials".

\section{Power calculation}

The trial aims to demonstrate that the rate of oral mucositis in the experimental group, $\pi_{E}$, is lower than the rate of oral mucositis in the control group, $\pi_{C}$. Hence, the trial aims to reject the one-sided null hypothesis $\mathrm{H}_{0}$ :

\begin{tabular}{|c|c|c|c|c|c|c|c|}
\hline \multirow[b]{3}{*}{ TIMEPOINT } & \multicolumn{6}{|c|}{ STUDY PERIOD } & \multirow{3}{*}{$\begin{array}{l}\text { Close-out } \\
t_{1+12 \text { months }} \\
\text { (lastfollow up) }\end{array}$} \\
\hline & \multirow{2}{*}{$\begin{array}{c}\text { Enrolment } \\
-t_{1}\end{array}$} & \multirow{2}{*}{$\begin{array}{c}\text { Allocation } \\
0 \\
0\end{array}$} & \multicolumn{4}{|c|}{ Treatment and follow up } & \\
\hline & & & $\begin{array}{c}\boldsymbol{t}_{1} \\
(\boldsymbol{R} T \text { start })\end{array}$ & $\begin{array}{c}t_{2} \\
(R T \text { end })\end{array}$ & $\begin{array}{c}t_{3} \\
(1 \quad \text { follow up 6-8 } \\
\text { weeks after } R T)\end{array}$ & $\begin{array}{c}\boldsymbol{t}_{4-x} \\
\text { (3-monthly } \\
\text { follow up) }\end{array}$ & \\
\hline ENROLMENT: & & & & & & & \\
\hline Eligibility screen & $\mathrm{X}$ & & & & & & \\
\hline Informed consent & $\mathrm{X}$ & & & & & & \\
\hline Allocation & & $\mathrm{X}$ & & & & & \\
\hline INTERVENTIONS: & & & & & & & \\
\hline Arm A: TRDs & & & & $\rightarrow$ & & & \\
\hline Arm B: Dental splints & & & $\leftarrow$ & $\rightarrow$ & & & \\
\hline ASSESSMENTS: & & & & & & & \\
\hline Clinical assessment & $\mathrm{X}$ & & & $\mathrm{X}$ & $\mathrm{X}$ & $\mathrm{X}$ & $\mathrm{X}$ \\
\hline Toxicity assessment & $\mathrm{X}$ & & & $\mathrm{X}$ & $\mathrm{X}$ & $\mathrm{X}$ & $\mathrm{X}$ \\
\hline QoL questionnaire & $\mathrm{X}$ & & & $\mathrm{X}$ & $\mathrm{X}$ & $\mathrm{X}$ & $\mathrm{X}$ \\
\hline Dental status & $\mathrm{X}$ & & & & $\mathrm{X}$ & & $\mathrm{X}$ \\
\hline CT/MRI head neck & $\mathrm{X}$ & & & & $\mathrm{X}$ & $\mathrm{X}$ & $\mathrm{X}$ \\
\hline
\end{tabular}

Fig. 2 Schedule of enrolment, interventions, and assessments for the prospective randomized controlled GUARD trial (SPIRIT figure). Abbreviations: Radiotherapy (RT), tissue retraction devices (TRDs), quality of life (QoL), computed tomography (CT), magnetic resonance imaging (MRI) 
$\pi_{E} \geq \pi_{C}$. We assumed that the rate of oral mucositis in certain anatomical regions in the experimental group will amount to $\pi_{\mathrm{E}}=15 \%$, while the rate of the control group is assumed to be $\pi_{E}=60 \%$. The assumed rate in the control group is based on published data $[4,5,27]$, while the rate in the experimental group reflects the assumption that the experimental intervention can achieve a decrease below grade III for the mucositis in the directly neighboring region of the tumor for $75 \%$ of all patients with mucositis. This hypothesis is based on preliminary clinical experience in ten patients treated with TRDs. Under these assumptions, 28 evaluable patients (14 per group) are required to achieve $80 \%$ power with a chi-square test at a one-sided 5\% level of significance. Assuming a 15\% dropout rate, 34 patients (17 per group) will be enrolled. The calculation of the sample size was performed using ADDPLAN v6.1.

\section{Analysis of the primary endpoint}

The null hypothesis $\pi_{E} \geq \pi_{C}$ will be evaluated at a onesided significance level of $5 \%$ using a chi-square test. Also, the associated odds ratio for the treatment effect will be provided with a one-sided $95 \%$ confidence interval. The analysis will be performed based on the safety population, including all randomized patients who were treated according to planned therapy for at least one day. Missing values for the primary outcome will be replaced by multiple imputations using the fully conditional specification method [27]. Sensitivity analyzes will be performed via complete-case analysis and the best and worst-case analysis.

\section{Analysis of secondary endpoints}

Secondary endpoints for OS and local control will be assessed using Kaplan Meier estimators. 1-year survival rates and median times of events will be provided with 95\% confidence intervals. Descriptive log-rank tests will be performed to compare the two treatment groups. All other secondary endpoints will be analyzed descriptively. The safety analysis will comprise a tabulation of absolute and relative frequencies for serious and adverse events, along with $95 \%$ confidence intervals.

The details of the statistical analysis will be defined in a statistical analysis plan (SAP) that will be finalized before the database lock. The analyzes will be performed with SAS v9.4 or higher.

\section{Ethics and safety considerations}

The Ethics Committee of Heidelberg University (S-394/2020) approved the study protocol, patient information form, and informed consent statement. The clinical trial will be conducted following the latest version of the "Declaration of Helsinki". Regarding the performance, evaluation, and documentation of this study, Good Clinical Practice (GCP) recommendations have been taken into consideration. The regulations concerning medical confidentiality and data protection are fulfilled. Adverse events will be monitored and recorded following GCP guidelines. The biocompatibility and stability of the FREEPRINT $^{\circledR}$ material used have already been approved for clinical application as a class IIa medical device. Therefore, a clinical investigation following the Medical Device Act does not apply to the current study.

\section{Discussion}

The primary aim of the current phase II study is to evaluate the efficacy of TRDs for the radiation treatment of head and neck tumors. The distance between the tumor and normal tissue can be increased in certain anatomical regions through the use of individualized TRDs, thus protecting the healthy oral mucosa from unwanted radiation exposure. We hypothesize that TRDs can significantly reduce the rate of side effects, as reducing treatment margins by a few millimeters can significantly attenuate treatment toxicity [14]. This could even reduce clinically relevant complications (e.g. weight loss due to painful swallowing) and long-term sequelae (e.g. xerostomia). Also, the jaw spacing and protrusion of the lower jaw by TRDs make breathing easier during treatment sessions, with consequences for patient comfort. However, it is not clear which approach is the most appropriate to assess the tolerability of treatment. Recently, PROs have gained importance in comparison with the conventional, physician-reported toxicity assessments, and quality of life questionnaires [26]. Therefore, in the current trial, the GRIX questionnaire and 12 selected PRO-CTC items relevant to patients with head and neck tumors will be used, besides the physician-reported toxicity profile.

Besides the primary objective of the trial, the present study will investigate several additional physical-technical and clinical aspects of individualized 3D printed TRDs. We hypothesize that the robustness of RT plans concerning positional variability of bone and soft tissue structures could be improved by TRD-mediated fixation. The degree of dose reduction in the oral mucosa by the use of TRD is currently unknown and will be assessed by plan comparisons between both treatment groups and correlated with clinical findings. Comparisons of in silico intrapatient RT plans, using previously available diagnostic CT/MRI scans, could reveal more functional aspects of TRDs. We will elaborate on these findings for various treatment approaches, including IMRT and particle therapy, as part of the study.

Oral evaluation and care before RT are well established for patients with HNC [19]. However, the longterm consequences on the dental condition are most 
likely underestimated. The present study aims to contribute to the understanding and prevention of dental sequelae after RT through systematic evaluations by a specialist in prosthetic dentistry.

The exploratory objective of the trial is to investigate gingival MSCs, derived from clinically indicated dental extractions tissue. We anticipate that the regenerative and radioprotective properties of MSCs could further improve the clinical tolerability of RT. However, the indirect tumor protective effects of MSCs must be ruled out before clinical use.

\section{Abbreviations}

CT: Computed tomography; CTC: Common Terminology Criteria; EORTC : European Organisation for Research and Treatment of Cancer; GCP: Good Clinical Practice; GRIX: Groningen Radiotherapy-Induced Xerostomia; HNC: Head and neck cancer; IMRT: Intensity-modulated radiotherapy; MRI: Magnetic resonance imaging; MSCs: Mesenchymal stem cells; PRO: Patient-reported outcomes; QLQ: Quality of Life Questionnaire; QUANTEC: Quantitative Analyses of Normal Tissue Effects in the Clinic; RIOM: Radiation-induced oral mucositis: RT: Radiotherapy; TRD: Tissue retraction device.

\section{Acknowledgements}

We thank our clinical trial center for the support of this study. We acknowledge Eric Tonndorf-Martini and Thomas Mielke for performing treatment planning and plan comparisons.

\section{Authors' contributions}

$\mathrm{TH}, \mathrm{CH}, \mathrm{FSS}, \mathrm{LC}$, and SA (principal investigator) developed and planned this trial under the supervision of $\mathrm{KH}, \mathrm{PR}$, and JD. TH, CH, FSS, LC, KL, SR, TE, JM, $\mathrm{JM}, \mathrm{KM}, \mathrm{KZ}, \mathrm{TM}, \mathrm{SH}, \mathrm{KH}, \mathrm{CF}$, and SA perform the patient treatment and clinical assessments. $\mathrm{TH}, \mathrm{SH}, \mathrm{KH}$, and SA perform treatment planning, dosimetry, and plan verification. JK is the trial statistician and responsible for statistical planning and statistical analysis. JK and SA are responsible for data management. $\mathrm{AH}$ and $\mathrm{CJ}$ coordinate the study. All authors read and approved the final manuscript.

\section{Funding}

Open Access funding enabled and organized by Projekt DEAL. The authors acknowledge financial support of the current study within the funding program of the National Center for Tumor Diseases (NCT) Heidelberg, Germany. Thomas Held and Sebastian Regnery are funded by the Physician-Scientist Program of Heidelberg University, Faculty of Medicine.

\section{Availability of data and materials}

Not applicable.

\section{Declarations}

\section{Ethics approval and consent to participate}

The Ethics Committee of the medical faculty of Heidelberg University approved this study on June 8th, 2020 (S-394/2020).

\section{Consent for publication}

Not applicable.

\section{Competing interests}

S.A. received grants from Novocure Inc., Accuray International Sàrl and Merck Serono GmbH outside the submitted work. J.D. received grants from CRI-The Clinical Research Institute GmbH, View Ray Inc., Accuray International Sàrl, Accuray Incorporated, RaySearch Laboratories AB, Vision RT limited, Merck Serono GmbH, Astellas Pharma GmbH, Astra Zeneca GmbH, Solution Akademie GmbH, Ergomed PLC Surrey Research Park, Siemens Healthcare GmbH, Quintiles $\mathrm{GmbH}$, Pharmaceutical Research Associates $\mathrm{GmbH}$, Boehringer Ingelheim Pharma GmbH Co, PTW-Freiburg Dr. Pychlau GmbH, Nanobiotix
A.A. outside the submitted work. The other authors declare no conflict of interest.

\section{Author details}

${ }^{1}$ Department of Radiation Oncology, Heidelberg University Hospital, Im Neuenheimer Feld 400, 69120 Heidelberg, Germany. ${ }^{2}$ Heidelberg Institute of Radiation Oncology (HIRO), Heidelberg, Germany. ${ }^{3}$ National Center for Tumor Diseases (NCT), Heidelberg, Germany. ${ }^{4}$ Clinical Cooperation Unit Radiation Oncology, German Cancer Research Center (DKFZ), Heidelberg, Germany. ${ }^{5}$ Heidelberg lon-Beam Therapy Center (HIT), Heidelberg, Germany. ${ }^{6}$ German Cancer Consortium (DKTK), partner site Heidelberg, German Cancer Research Center (DKFZ), Heidelberg, Germany. ${ }^{7}$ Department of Prosthetic Dentistry, Heidelberg University Hospital, Heidelberg, Germany. ${ }^{8}$ Institute of Medical Biometry and Informatics (IMBI), Heidelberg University Hospital, Heidelberg, Germany. ${ }^{9}$ Department of Oral and Maxillofacial Surgery, University Hospital Heidelberg, Heidelberg, Germany. ${ }^{10}$ Department of Otorhinolaryngology, University of Heidelberg, Heidelberg, Germany.

Received: 5 January 2021 Accepted: 7 April 2021

Published online: 17 April 2021

\section{References}

1. Cooper JS, et al. Postoperative concurrent radiotherapy and chemotherapy for high-risk squamous-cell carcinoma of the head and neck. N Engl J Med. 2004;350(19):1937-44.

2. Bernier J, et al. Postoperative irradiation with or without concomitant chemotherapy for locally advanced head and neck cancer. N Engl J Med. 2004;350(19):1945-52.

3. Maria OM, Eliopoulos N, Muanza T. Radiation-induced oral mucositis the delete section. Front Oncol. 2017;7(89):1-23.

4. Elting LS, et al. Risk, outcomes, and costs of radiation-induced oral mucositis among patients with head-and-neck malignancies. Int J Radiat Oncol Biol Phys. 2007;68(4):1110-20.

5. Bonner JA, et al. Radiotherapy plus cetuximab for locoregionally advanced head and neck cancer: 5 -year survival data from a phase 3 randomized trial, and the relation between cetuximab-induced rash and survival. Lancet Oncol. 2010;11(1):21-8.

6. Naidu MUR, et al. Chemotherapy-induced and/or radiation therapyinduced oral mucositis - complicating the treatment of cancer. Neoplasia. 2004;6(5):423-31.

7. Nutting $\mathrm{CM}$, et al. Parotid-sparing intensity modulated versus conventional radiotherapy in head and neck cancer (PARSPORT): a phase 3 multicentre randomised controlled trial. Lancet Oncol. 2011;12(2):127-36.

8. Spencer $\mathrm{CR}$, et al. Eliminating radiotherapy to the contralateral retropharyngeal and high-level II lymph nodes in head and neck squamous cell carcinoma is safe and improves the quality of life. Cancer. 2014;120(24):3994-4002.

9. Muanza TM, et al. Evaluation of radiation-induced oral mucositis by optical coherence tomography. Clin Cancer Res. 2005;11(14):5121-7.

10. Bodard AG, et al. A new, simple maxillary-sparing tongue depressor for external mandibular radiotherapy: a case report. Head Neck. 2009:31(11):1528-30.

11. Ikawa $\mathrm{H}$, et al. A custom-made mouthpiece incorporating tongue depressors and elevators to reduce radiation-induced tongue mucositis during carbon-ion radiation therapy for head and neck cancer. Pract Radiat Oncol. 2018;8(2):e27-31.

12. Ikawa $\mathrm{H}$, et al. The efficacy of a custom-made mouthpiece with spacer to reduce osteoradionecrosis in carbon-ion radiation therapy for tonguebase tumor. Adv Radiat Oncol. 2018;4(1):15-9.

13. Inoue $Y$, et al. Are intraoral stents effective for reducing the severity of oral mucositis during radiotherapy for maxillary and nasal cavity cancer? J Oral Maxillofac Surg. 2020;78(7):1214.e1-1214.e8.

14. Navran A, et al. The impact of margin reduction on outcome and toxicity in head and neck cancer patients treated with image-guided volumetric modulated arc therapy (VMAT). Radiother Oncol. 2019;130:25-31.

15. Nguyen CT, Lee VS, Wu J. An acrylic immobilization bite block for use during radiation therapy: description of a new technique. Int J Prosthodont. 2018;31(4):338-41. 
16. Waldecker $M$, et al. Fully digital fabrication of an occlusal device using an intraoral scanner and 3D printing: a dental technique. J Prosthet Dent. 2019;121(4):576-80.

17. Schwindling FS, et al. Three-dimensional-guided removal and preparation of dental root posts-concept and feasibility. J Prosthodont Res. 2020;64(1):104-8.

18. Nicolay $\mathrm{NH}$, et al. Mesenchymal stem cells - a new hope for radiotherapy-induced tissue damage? Cancer Lett. 2015;366(2):133-40.

19. Koga DH, Salvajoli JV, Alves FA. Dental extractions and radiotherapy in head and neck oncology: review of the literature. Oral Dis. 2008;14(1):40-4.

20. Marks LB, et al. Use of normal tissue complication probability models in the clinic. Int J Radiat Oncol Biol Phys. 2010;76(3 Suppl):S10-9.

21. Bentzen SM, et al. Quantitative Analyses of Normal Tissue Effects in the Clinic (QUANTEC): an introduction to the scientific issues. Int J Radiat Oncol Biol Phys. 2010;76(3 Suppl):S3-9.

22. Grégoire $V$, et al. Delineation of the primary tumor Clinical Target Volumes (CTV-P) in laryngeal, hypopharyngeal, oropharyngeal and oral cavity squamous cell carcinoma: AIRO, CACA, DAHANCA, EORTC, GEORCC, GORTEC, HKNPCSG, HNCIG, IAG-KHT, LPRHHT, NCIC CTG, NCRI, NRG Oncology, PHNS, SBRT, SOMERA, SRO, SSHNO, TROG consensus guidelines. Radiother Oncol. 2018;126(1):3-24.

23. Biau J, et al. Selection of lymph node target volumes for definitive head and neck radiation therapy: a 2019 Update. Radiother Oncol. 2019;134:1-9.
24. Bjordal K, et al. Development of a European Organization for Research and Treatment of Cancer (EORTC) questionnaire module to be used in quality of life assessments in head and neck cancer patients EORTC Quality of Life Study Group. Acta Oncol. 1994;33(8):879-85.

25. Beetz I, et al. The Groningen Radiotherapy-Induced Xerostomia questionnaire: development and validation of a new questionnaire. Radiother Oncol. 2010;97(1):127-31.

26. Kluetz PG, et al. Patient-reported outcomes in cancer clinical trials: measuring symptomatic adverse events with the National Cancer Institute's Patient-Reported Outcomes Version of the Common Terminology Criteria for Adverse Events (PRO-CTCAE). Am Soc Clin Oncol Educ Book. 2016:35:67-73

27. van Buuren S. Multiple imputations of discrete and continuous data by the fully conditional specification. Stat Methods Med Res. 2007;16(3):219-42.

\section{Publisher's Note}

Springer Nature remains neutral with regard to jurisdictional claims in published maps and institutional affiliations.
Ready to submit your research? Choose BMC and benefit from:

- fast, convenient online submission

- thorough peer review by experienced researchers in your field

- rapid publication on acceptance

- support for research data, including large and complex data types

- gold Open Access which fosters wider collaboration and increased citations

- maximum visibility for your research: over $100 \mathrm{M}$ website views per year

At BMC, research is always in progress.

Learn more biomedcentral.com/submissions 